KINDHEIT IN DEUTSCHLAND AUS DER PERSPEKTIVE NEU ZUGEWANDERTER KINDER: ERFAHRUNGEN, DEUTUNGEN, STRATEGIEN

Jessica Schwittek

Universität Duisburg-Essen, Institut für Erziehungswissenschaft

E-Mail: jessica.schwittek@uni-due.de

URL: https://www.uni-due.de/biwi/koenig/schwittek.php

Zitationsvorschlag:

Schwittek, Jessica (2021): Kindheit in Deutschland aus der Perspektive neu zugewanderter Kinder: Erfahrungen, Deutungen, Strategien. In: Gesellschaft - Individuum - Sozialisation (GISO). Zeitschrift für Sozialisationsforschung, 2 (1). DOI:

10.26043/GISo.2021.1.1

Link zum Artikel:

https://doi.org/10.26043/GISo.2021.1.1 


\title{
KINDHEIT IN DEUTSCHLAND AUS DER PERSPEKTIVE NEU ZUGEWANDERTER KINDER: ERFAHRUNGEN, DEUTUNGEN, STRATEGIEN
}

\author{
Jessica Schwittek
}

\begin{abstract}
Der Beitrag interessiert sich für den Blick neu zugewanderter Kinder auf Kindheit in Deutschland und geht der Frage nach, wie diese mit einer ihnen - zunächst - fremden Kindheit umgehen. Auf der Grundlage von Gruppendiskussionen wird herausgearbeitet, dass die Teilnehmerlnnen Kindheit in Deutschland vor dem Hintergrund von (Sozialisations-) Erfahrungen in den Herkunftsländern als veranstaltete Kindheit und als (relativ) sichere Kindheit deuten. Diese beiden Deutungs- und Erfahrungskomplexe sowie die von den Kindern entworfenen Handlungsstrategien werden aus dem empirischen Material heraus rekonstruiert. Deutlich werden so die kompetente Ermittlung und Anwendung sozialer Regeln durch die Akteurlnnen und ihre kreativen Leistungen hinsichtlich des Umgangs mit neuen Situationen.
\end{abstract}

Keywords: Neu zugewanderte Kinder, Kindheit, Interpretation der Situation, Erfahrungen, Strategien, Gruppendiskussionen

\section{EINFÜHRUNG}

Der Beitrag geht der Frage nach, wie Kinder, die kürzlich aus Ländern des Globalen Südens nach Deutschland migriert sind, auf Kindheit in Deutschland schauen und welche Strategien sie im Umgang mit einer innen zunächst fremden Kindheit entwerfen. ${ }^{1}$ Verfolgt wird damit einerseits das kindheitssoziologische Interesse, Kindheit aus der Perspektive von Kindern selbst zu beleuchten. Andererseits soll die bisherige Forschungslage zur Gruppe neu zugewanderter Kinder erweitert werden, indem vertiefte Einblicke in die Bedingungen und Herausforderungen ihres Integrationsprozesses gewonnen werden.

Ausgangpunkt der hier vorgestellten Analyse ist das sozialtheoretische Verständnis von Kindheit als eine Struktur gesellschaftlicher Ordnung (Ala- nen 2005) und, daran anschließend, der empirischen Variabilität von Kindheit in unterschiedlichen Gesellschaften (Bühler-Niederberger 2020a). Damit verknüpft ist die Annahme, dass transnational migrierende Kinder aus Ländern des Globalen Südens in eine Kindheit eintreten, die potenziell anders strukturiert ist als diejenige in ihren Herkunftsländern. Insbesondere für junge Menschen, die aus Kriegs- und Krisengebieten oder aufgrund von ökonomischer Not nach Deutschland migriert sind, ist zu erwarten, dass ihre Kindheit in den Herkunftsländern wenig gemeinsam hat mit der "langen und behüteten Kindheit" (ebd.), wie sie für Deutschland postuliert werden kann (Zito 2017, 238). Aus der Subjektperspektive können diese unterschiedlichen Erfahrungen bzw. die Neuheit kindlicher Lebenswelten, wie neu zugewanderte Kinder sie in Deutschland vorfinden, zu Erlebnissen der Irritation und, daran anschließend, zu Prozessen der

\footnotetext{
1 Zur Klärung dieses Begriffs: Gemeint sind Kinder, die innerhalb der letzten zwei Jahre vor der Datenerhebung, welche zwischen März 2018 und Juni 2019 stattfand, nach Deutschland migriert sind. Die (nicht unproblematische) Kategorie des Globalen Südens wird herangezogen, um Länder zu bezeichnen, die sich sowohl hinsichtlich ihrer wirtschaftlichen Lage als auch in ihren gesellschaftlichen Strukturen (insbesondere solche, die für das Aufwachsen relevant sind) substantiell von Deutschland unterscheiden (vgl. Kapitel 2.2). Keine Spezifizierung wird im vorliegenden Beitrag hinsichtlich der Art der Migration vorgenommen. Das Sample beinhaltet sowohl geflüchtete Kinder als auch solche, die aus europäischen Ländern außerhalb der EU, im Zuge des europäischen Freizügigkeitsprinzips oder im Rahmen von Familienzusammenführungen nachgezogen sind. Eine ähnliche Gruppenkonstruktion ist bspw. auch bei Massumi/von Dewitz (2015, 11 ff.) zu finden.
} 
Reflexion führen. Diese neuen Situationen verlangen danach, von den beteiligten AkteurInnen gedeutet zu werden, um sich in der neuen Lebenswelt zurechtzufinden und sich darin zu positionieren. Einem interpretativen Paradigma folgend, wird davon ausgegangen, dass die Deutungen sozialer Welt und das eigene Handeln darin immer auf der Grundlage bisheriger Erfahrungen stattfinden (Blumer 1969). Übertragen auf den hier untersuchten Kontext heißt dies, dass neu zugewanderte Kinder die Deutung von Kindheit in Deutschland und die Strategien ihres Umgangs damit auf Basis ihrer bisherigen Erfahrungen vornehmen. Wie genau sie dies tun und welche Bedeutung dabei ihren Erfahrungen aus den Herkunftsländern zukommt, wird als empirisch zu beantwortende Frage gestellt. Die hier vorgestellte Analyse interessiert sich damit a) für die Irritationen, die neu zugewanderte Kinder angesichts von Kindheit in Deutschland erleben, b) dafür, welche Erfahrungen aus dem Herkunftsland dabei relevant sind bzw. relevant gemacht werden, und c) für die Strategien, die sie aus den jeweils neuen Situationsdeutungen für sich entwerfen.

Damit wird eine dezidiert prozesshafte Perspektive auf das Ankommen neu zugewanderter Kinder eingenommen, die sowohl die heterogenen Erfahrungskomplexe (Thomas 1926) dieser Gruppe einschließt, als auch die mehrdimensionalen Herausforderungen ihrer Lebenssituation in Deutschland berücksichtigt und diese als ein komplexes Ineinandergreifen versteht.

Um diesen Erkenntnisinteressen nachzugehen, wurden Gruppendiskussionen mit insgesamt 35 neu zugewanderten Kindern im Alter zwischen acht und 15 Jahren geführt. ${ }^{2}$ Fokus der Gruppendiskussionen war das Thema Kindheit in Deutschland, für das die Teilnehmerlnnen in dem Sinne als ExpertInnen angesprochen wurden, als dass sie eigene (Sozialisations-)Erfahrungen in
Deutschland sowie in (mindestens) einem anderen Land gemacht haben und daher über Wissen hinsichtlich der Unterschiede und Gemeinsamkeiten unterschiedlich strukturierter Kindheiten verfügen. $^{3}$

Aus dem Material wurden zwei „Deutungs- und Erfahrungskomplexe" und drei Strategien rekonstruiert, die Prozesse der Verknüpfung von früheren Erfahrungen mit den situativen Deutungen von Kindheit in Deutschland beschreiben. Dabei handelt es sich um die Deutungs- und Erfahrungskomplexe von Kindheit in Deutschland 1. als eine veranstaltete und 2. als eine (relativ) sichere Kindheit. In den drei Strategien werden unterschiedliche Weisen des Umgangs mit der "veranstalteten und (relativ) sicheren Kindheit" rekonstruiert, die in unterschiedlichem Maße als Formen der Gefügigkeit oder der kreativen Gestaltung der eigenen Lebenswelt gelesen werden können.

\section{NEU ZUGEWANDERTE KINDER IN IHREN HERKUNFTS- UND ANKUNFTSKONTEXTEN}

In diesem Kapitel werden kursorisch einige zentrale Befunde skizziert, die die Flucht- und Migrationsforschung sowie die sozialwissenschaftliche Kindheitsforschung hinsichtlich der Erfahrung von neu zugewanderten Kindern in Deutschland und hinsichtlich der Kindheit in Ländern des Globalen Südens herausgearbeitet haben. ${ }^{4}$

\subsection{Neu zugewanderte Kinder in Deutschland}

Studien, die die Situation neu zugewanderter Kinder im Ankunftskontext Deutschland untersuchen, betrachten diese häufig in ihrer Rolle als Schülerlnnen und fokussieren Fragen der Partizipation im Bildungssystem. Hier werden systematische Benachteiligungen neu zugewanderter Kinder hinsichtlich der besuchten Schulform (El-

\footnotetext{
2 Je nach Kategorisierung dieser Altersspanne könnte hier auch von Kindern und Adoleszenten gesprochen werden. Aufgrund der kindheitstheoretischen Einbettung des Beitrags wird aber auf eine Hinzunahme des Adoleszenzbegriffs verzichtet, obgleich sich hierdurch interessante weiterführende Fragestellungen zum „adoleszenten Möglichkeitsraum“ im Kontext der Migration anschließen ließen (Günther et al. 2010).

${ }^{3}$ Man könnte auch von einem „ethnographischen“ oder „fremden“ Blick sprechen (Agar 2006), den neu zugewanderte Kinder (zunächst) auf Kindheit in Deutschland haben; eine Perspektive, die sich sowohl von der Perspektive Erwachsener als auch von derjenigen nicht migrierter Kinder unterscheidet.

${ }^{4}$ Im Folgenden werden keine Befunde zur Situation von unbegleiteten Minderjährigen aufgeführt, da diese Gruppe in der vorliegenden Studie nicht untersucht wurde.
} 
Mafaalani/Kemper 2017), durch segregierte Beschulungsmodelle (Brüggemann/Nikolai 2016) sowie durch ausgrenzende und strukturell diskriminierende Prozedere von Behörden und Institutionen problematisiert (Eisenhuth 2015; Emmerich et al. 2017; Karakayali 2018). Gleichzeitig starten junge Geflüchtete mit vergleichsweise hohen Bildungsaspirationen (Bohn et al. 2016, 17) und erfahren diesbezüglich Druck von Seiten der Familien, aber auch von BetreuerInnen und Lehrerlnnen (Lechner/Huber 2017, 65 ff.).

Die Perspektive der Kinder selbst findet insgesamt wenig Berücksichtigung (Gerarts/Andresen 2018, 162), einige Studien geben aber Hinweise auf die große Bedeutung sozialer Einbindung und den ausgeprägten Wunsch, Freunde zu finden. Neu zugewanderte Schülerlnnen bemängeln die segregierten Beschulungsformen und Lebenswelten (Bohn et al. 2016), die den Kontakt zu deutschen Gleichaltrigen erschweren oder sogar verhindern (Gurski/Rother 2018). Gleichwohl ist der Kontakt zu den einheimischen Peers auch mit Ambivalenzen verbunden, bspw. aufgrund erlebter oder befürchteter Ausgrenzungen und rassistischer Diskriminierungen (Diekmann/Fereidooni 2019, 352 f.). So deuten Studien darauf hin, dass junge neu zugewanderte Menschen mitunter Formen des Stigmamanagements (Goffman 2016) betreiben, dass sie bspw. ihren Flüchtlingsstatus verschweigen (Lechner/Huber 2017, 108) oder bestimmte, bspw. religiöse, Symbole ablegen (Bohn et al. 2016). Diese Strategien können als Versuch verstanden werden, Anzeichen eines natio-ethno-kulturellen Andersseins (Mecheril 2002) zu verstecken und sich damit als normal hinsichtlich hegemonialer Diskurse zu positionieren.

Die familialen Beziehungen werden unter dem Stichwort unterschiedlicher Akkulturationsgeschwindigkeiten zwischen Eltern und deren Kindern in Zuwandererfamilien thematisiert (Titzmann/Juang 2018). Dadurch kann eine (extensive) Inanspruchnahme von Kindern als Dolmetscherlnnen oder anderweitig als Sorgende für Familienangehörige (bspw. psychisch belastete Eltern) entstehen. Diese stehen potentiell in Konkurrenz zu schulischen und peerbezogenen Interessen der Kinder, aber auch in Kontrast zu normativen Vorstellungen von Kindheit in Deutschland (Wihstutz/Schiwarov 2018). ${ }^{5}$

Ein weiterer Schwerpunkt hinsichtlich der Erfahrung von Kindern bzw. Jugendlichen aus Migrantenfamilien, der aber weitgehend in Studien zur zweiten bzw. dritten Generation sog. GastarbeiterInnen herausgearbeitet wurde, bezieht sich auf Differenzerfahrungen zwischen unterschiedlichen Relevanzsystemen: demjenigen der Familie bzw. der ethnischen Community, welche von der Kultur des Herkunftslandes (der Eltern bzw. Großeltern) geprägt ist, und derjenigen der Mehrheitsgesellschaft, vermittelt in erster Linie durch die Schule und die Peergroup. Diese Differenzerfahrung wird als normativer Bruch zwischen der sog. "inneren Sphäre" und der „äußeren Sphäre" erlebt (Bohnsack 2003; Nohl 2001). Laut El-Mafaalani ist die innere Sphäre durch eine traditionelle und kollektivistische Orientierung sowie eine hohe Bedeutung von Ehre, Respekt und Liebe gekennzeichnet, die äußere Sphäre durch Selbstbezüglichkeit, Loyalität und implizite Normen, die es mühsam zu entziffern gilt $(2013,115$ ff.). Die Sphärendifferenz als spezifischer Erfahrungsraum junger MigrantInnen ist bereits in der Kindheit von hoher Relevanz (ebd.) und Kinder positionieren sich darin als irritierte und unsichere oder aber als planvolle Akteurlnnen (Kämpfe 2019, 147 ff.).

Die Befunde zu den Differenzerfahrungen von Kindern, deren Familien bereits seit geraumer Zeit in Deutschland leben, sind nur begrenzt auf Kinder übertragbar, die selbst vor kurzer Zeit nach Deutschland migriert sind. Gleichwohl stellen sie gewinnbringende Anknüpfungspunkte für die vorliegende Studie dar. Aufgegriffen wird der Aspekt divergierender Erfahrungen, wobei aber weniger auf die Differenzen zwischen der inneren und der äußeren Sphäre fokussiert wird, sondern eher auf die Erfahrungen der Kinder in unterschiedlichen (biographischen) Episoden: derjenigen in der Herkunfts- und derjenigen der

\footnotetext{
${ }^{5}$ Im angelsächsischen Raum wird eine weitaus lebendigere öffentliche und wissenschaftliche Debatte zum „young caring and language brokering" (Crafter et al. 2017) geführt, in der auch Kinder aus Zuwandererfamilien explizit in den Blick genommen werden (Bauer 2016).
} 
Ankunftsgesellschaft. Die Migration der Kinder wird damit als biographische Zäsur verstanden, die den Wechsel von der einen in eine anders strukturierte Kindheit bzw. generationale Ordnung und die damit jeweils zusammenhängenden, oft impliziten Regeln und Normalitätsvorstellungen markiert. ${ }^{6}$

\subsection{Kindheiten im Globalen Süden}

Aufgrund der für die vorliegenden Studie weit gefassten Gruppe neu zugewanderter Kinder ist es nicht einmal ansatzweise möglich, einen Überblick über die heterogenen Lebens- und Aufwachsensbedingungen in den Herkunftsländern zu geben. Im Folgenden werden daher nur holzschnittartig einige Themenfelder benannt, die die (sozialwissenschaftliche) Kindheitsforschung zur Beschreibung von Kindheiten im Globalen Süden bzw. im Vergleich mit solchen des Globalen Nordens herausgearbeitet hat.?

Zum einen betrifft dies die ursprüngliche Unterscheidungsdimension der wirtschaftlichen Lage bzw. des „Entwicklungsstandes“ der Länder, die der Kategorisierung durch internationale Organisationen zugrunde liegt. Sie wirkt sich in vielfacher Weise auf Kindheit aus, bspw. hinsichtlich Überlebensraten, Gesundheit, frühkindlicher Entwicklung, Zugang zu Bildung, Legitimität und Aufkommen von Gewalt gegen Frauen und Kinder, sozialer Sicherungssysteme und zahlreicher weiterer Indikatoren, wie sie bspw. durch die Reporte von UNICEF abgebildet werden (bspw. die „State of the World's Children“-Reporte oder die MICS-Surveys). Insbesondere in Kriegs- und Krisengebieten ist der Schutz vor Gewalt für Kinder nicht gegeben; psychologischen Studien zufolge berichtet ca. ein Drittel syrischer Kinder im Schulalter über traumatisierende Erfahrungen und leidet an Traumafolgestörungen (Perkins et al. 2018). Zu ähnlichen Einschätzungen kommen Studien zu Flüchtlingskindern in Deutschland
(Soykoek et al. 2017). Qualitative Studien zeigen, dass das mitunter extreme Maß sozialer Ungleichheit in Ländern des Globalen Südens fundamental unterschiedliche Kindheiten hervorbringen kann (Bourdillon/Boyden 2014) mitunter in ein und demselben Haushalt (Jaquemin 2004).

Forschung, die an das Begriffspaar „kollektivistisch - individualistisch" aus der kulturvergleichenden Psychologie angelehnt ist (Hofstede 1980), hat Befunde zu independenten bzw. interdependenten Orientierungen vorgelegt, die sich in den privaten und institutionellen Sozialisations- und Erziehungspraktiken niederschlägt (Kagitcibasi 1996) und bereits ab dem Säuglings- und Kleinkindalter in die Gestaltung intergenerationaler Beziehungen einfließt (Keller 2007). Ländern des Globalen Südens werden (eher) kollektivistische Orientierungen zugeschrieben und eine Ausrichtung der Erziehung auf ein relationales Selbst (Kitayama 2002). Hervorgehoben wird die Funktionalität für die Familie bereits in jungem Alter und Gehorsam gegenüber den Eltern bzw. allgemein des Respekts gegenüber Erwachsenen. Kinder erleben eine starke Verbundenheit mit der Familie, aber auch hohen Konformitätsdruck und ein ausgeprägtes Verpflichtungsgefühl (Bühler-Niederberger/Schwittek 2014).

Die Gestaltung intergenerationaler Beziehungen ist vor dem Hintergrund gesamtgesellschaftlicher generationaler Solidaritätsmuster zu verstehen, welche sehr unterschiedlich ausfallen können. In Abhängigkeit vom Vorhandensein wohlfahrtsstaatlicher Strukturen wird die Versorgung und Sicherung des Einzelnen vorrangig durch öffentliche Unterstützungssysteme oder aber durch die privaten Kollektive geleistet (Bühler-Niederberger 2020b). Letztere Konstellation kann mit ausgeprägten interpersonellen Abhän-

\footnotetext{
${ }^{6}$ Dabei ist wichtig anzumerken, dass es sich hier um eine analytische Trennung handelt, die einen verhältnismäßig starren Strukturbegriff anlegt. Der Autorin ist bewusst, dass die gelebte Wirklichkeit von Migrantlnnen sich durch eine Multiplexität nationaler, ethnischer und kultureller Bezüge auszeichnet und zunehmend in transnationalen sozialen Räumen verortet ist (vgl. dazu auch Twum-Danso Imoh et al. 2019). Die Verknüpfung unterschiedlicher Referenzrahmen wird in der vorliegenden Studie vor allem durch die Analyse der von den Kindern vorgenommenen Situationsdeutungen eingeholt, in denen sowohl vergangene (Sozialisations-) Erfahrungen relevant gemacht als auch Strategien für Gegenwart und Zukunft entworfen werden.

7 Für eine Einordnung und die Herkunft der Unterscheidung von "Global South“ und "Global North" siehe Dados/Connell (2012).
} 
gigkeiten entlang der Kategorien Alter und Geschlecht in innerfamilialen Ordnungsarrangements einhergehen.

An dieser Stelle soll noch einmal betont werden, dass es sich bei den hier skizzierten Unterscheidungen (Entwicklungsstand, individualistisch/kollektivistisch, independent/interdependent) nicht um dichotome Kategorien handelt, sondern eher um Kontinuen, in denen sich die vielfältigen Mischformen, wie sie in den (wiederum heterogenen) nationalen bzw. gesellschaftlichen Kontexten vorkommen, einordnen lassen. Sie dienen dazu, Richtungen anzudeuten, in denen mögliche Unterschiede zwischen den „hier" und "dort" erlebten Kindheiten neu zugewanderter Kinder liegen können. Absichtlich werden keine konkreten Vorannahmen über die (Sozialisations-)Erfahrungen formuliert, die neu zugewanderte Kinder in den Herkunftsländern gemacht haben könnten; vielmehr wurde die Frage nach deren Art und Bedeutung empirisch bearbeitet. Gefragt wird, welche Unterschiede zwischen der Kindheit im Herkunftsland und derjenigen in Deutschland überhaupt wahrgenommen werden und welche konkreten Erfahrungen die Kinder dabei als relevant einschätzen.

\section{ERKENNTNISINTERESSE UND AUFBAU DER STUDIE}

Der Beitrag folgt einem kindheitssoziologischen Blick mit dem Standpunkt, dass Kindheit gesellschaftsabhängig ist (Qvortrup 2005). Dies bedeutet zum einen, dass die Art und Weise, wie Kindheit strukturiert ist, eng mit der gesamtgesellschaftlichen Ordnung verbunden ist und dass unterschiedliche Gesellschaften auch durch unterschiedliche Kindheiten charakterisiert sind. Zum anderen bedeutet es, dass konkrete, gelebte Kindheiten ganz maßgeblich von den jeweiligen generationalen Ordnungsarrangements abhängen, an denen Kinder als Komplizen mitwirken (Bühler-Niederberger 2020a, 237). Kinder, und insbesondere migrierende Kinder, sind darüber hinaus mit „variierenden Regelsets“ und mitunter "normativen Brüchen“ (ebd., 257) konfrontiert, die sie zu bearbeiten haben. Vor diesen grundlegenden Annahmen werden neu zugewanderte Kinder als eine Gruppe entworfen, die Sozialisationserfahrungen in anders strukturier- ten Gesellschaften gemacht hat, und sich, angesichts ihrer Ankunft in Deutschland, mit neuen Regelsets vertraut macht. Das zentrale Erkenntnisinteresse der Studie betrifft die Frage, wie neu zugewanderte Kinder - vor dem Hintergrund ihrer Erfahrungen in Gesellschaften des Globalen Südens - Kindheit in Deutschland deuten und welche Handlungslinien sie daraus entwickeln. Dahinter steht einerseits das (kindheits)soziologische Interesse an der Variabilität von Kindheit, die hier aus der Perspektive der Kinder selbst rekonstruiert wird. Die untersuchte Gruppe ist soziologisch gesehen vor allem deswegen interessant, weil sie Aufschluss über die AkteurInnenschaft von Kindern angesichts der Bewältigung normativer Brüche geben kann. Aus einer gesellschaftlichen Perspektive ist die Untersuchung relevant, weil über die rekonstruierten Irritationen und Deutungen Erkenntnisse sowohl zu den Hürden als auch zu den Gelingensbedingungen von Integrationsprozessen neu zugewanderter Kinder gewonnen werden.

Für die hier präsentierte Analyse wird Migration als ein Geschehen verstanden, das die migrierenden Kinder vor eine Vielzahl neuer Situationen stellt, in denen die in früheren Interaktionen erworbenen Deutungsschemata (möglicherweise oder teilweise) nicht mehr zielführend sind (Oerter 2013). Dies stellt die AkteurInnen vor die Herausforderung, die neuen Situationen zu deuten, um darüber zu neuen, besser auf die Situation abgestimmten Strategien zur Verfolgung der eigenen Ziele (bspw. Bildungsziele oder der Anschluss an die Peergroup) zu gelangen. Die Deutung von Situationen, so die grundlegende Annahme des symbolischen Interaktionismus, geschieht dabei auf der Grundlage von Bedeutungen, die in bereits zurückliegenden Interaktionen entwickelt wurden (Blumer 1969). Aus einer solch interaktionistischen Perspektive wird also davon ausgegangen, dass neu zugewanderte Kinder sich ihre Kindheit in Deutschland auf der Grundlage von Bedeutungen erschließen, die sie in Interaktionskontexten ihrer Kindheit in den Herkunfts- und Transitländern erworben haben. Thomas $(1926,40)$ spricht von „Erfahrungskomplexen", in denen sich thematisch bzw. kontextuell zusammenhängende Erfahrungen kondensieren und die zu Deutungsfolien neuer Situationen werden (können). Der Begriff des Erfahrungskomplexes wird für die vorliegende 
Analyse zum Begriff des „Deutungs- und Erfahrungskomplexes" erweitert. Betont wird damit der Prozess des transsituativen Verknüpfens vergangener Erfahrungen und neuer Situationen durch die Akteurlnnen.

\subsection{Methodischer Zugang}

Die vorliegende Analyse beruht auf den Daten einer multimethodisch angelegten Pilotstudie zu den sozialen Welten neu zugewanderter Kinder in Deutschland. Neun Gruppendiskussionen wurden mit insgesamt 35 Kindern geführt, von denen 17 in einer zweiten Erhebungsphase in Einzelinterviews zu ihren persönlichen Netzwerken familialer und freundschaftlicher Beziehungen sowie Perspektiven und Wünschen für die Zukunft befragt wurden. Die hier vorgestellte Analyse bezieht sich lediglich auf die Gruppendiskussionen.

Befragt wurden neu zugewanderte Kinder und Adoleszente im Alter zwischen neun und $15 \mathrm{Jah}-$ ren, die zum Zeitpunkt der Datenerhebung zwischen ein und zwei Jahren in Deutschland lebten. Als Herkunftsländer wurden genannt: Ägypten, Bosnien, Irak, Iran, Marokko, Moldawien, Rumänien, Serbien, Syrien. Alle befragten Kinder migrierten mit einem oder mehreren Familienangehörigen nach Deutschland. ${ }^{8}$ Der Zugang zu den Kindern erfolgte über Kontakte zu Lehrkräften sowie über ehrenamtlich tätige Kontaktpersonen in mehreren Großstädten in Nordrhein-Westfalen. Die Samplingstrategie zielte auf eine möglichst hohe Varianz in Hinblick auf unterschiedliche Kriterien wie Alter, Wohnsituation und Schulform. Befragt wurden die Kinder als Expertlnnen für ihr eigenes Leben (Heinzel 2000). Darüber hinaus werden sie in den hier präsentierten Gruppendiskussionen aber auch als ExpertInnen für Kindheit als eine soziale (generationale) Struktur angesprochen, da sie diese in unterschiedlichen Ausgestaltungen als eigene Sozialisationskontexte erfahren haben und direkte Vergleiche ziehen können.
Die Gruppendiskussionen wurden als ein Instrument der Datenerhebung gewählt, welches es ermöglicht, das konjunktive Wissen (Bohnsack 2012) der Befragten herauszuarbeiten. Das Vorgehen wurde folgendermaßen gestaltet: Die jeweilige Kontaktperson bzw. Lehrkraft lud die Forscherlnnen zu einem Kennenlernen mit den Kindern ein. Anschließend wurden Termine für die Gruppendiskussionen verabredet, welche in Räumlichkeiten der Schulen stattfanden. Hier diente bei den jüngeren Kindern eine Bildergeschichte als Stimulus, die eine Migration/Reise und das Ankommen in einem neuen Kontext zum Gegenstand hatte. Nach der gemeinsamen Betrachtung der Bilder wurden die Kinder eingeladen, über die Geschichte zu reflektieren und ihre Eindrücke dazu zu schildern. Die Gruppendiskussionen mit den älteren Kindern (ab 12 Jahren) wurden mit einem Erzählstimulus eingeleitet: Sie wurden eingeladen, sich vorzustellen, dass eine befreundete Person, die selbst noch nie in Deutschland gewesen ist, sie nach ihren Eindrücken von Kindheit in Deutschland fragen würde. Angestrebt wurde ein möglichst selbstläufiges Gespräch unter den Teilnehmerlnnen, was in der Umsetzung aber nur episodenweise gelang. Häufig wurden initiierende und zum weiteren Erzählen anregende Nachfragen durch die ModeratorInnen gestellt, auf die die Kinder (der Reihe nach) antworteten, anstatt untereinander ins Gespräch zu kommen. Dies ist möglicherweise auf die Ungewohntheit der Gesprächssituation für die Teilnehmerlnnen zurückzuführen, bzw. auf den schulischen Kontext und auf Unterrichtssituationen, die sich am Frage-Antwort-Verfahren orientieren.

\subsection{Datenanalyse}

Die Gruppendiskussionen wurden aufgezeichnet, verbatim transkribiert und anschließend zunächst in einem offenen und intuitiven Kodierdurchgang erschlossen (Strauss/Corbin 1990). Als sensibilisierendes, an den skizzierten Theorien orientiertes Konzept (sensu Blumer)

\footnotetext{
8 Unbegleitete Minderjährige wurden nicht absichtlich aus der Stichprobe ausgeschlossen. Vielmehr wurden über unsere Kontakte ins Feld keine Personen aus dieser Gruppe erreicht. Es kann davon ausgegangen werden, dass dies an der geringen Anzahl von unbegleiteten Minderjährigen in der hier interessierenden Altersgruppe liegt. Der Großteil unbegleiteter minderjähriger Geflüchteter in Deutschland ist 16 Jahre alt oder älter (Tangermann/Hoffmeyer-Zlotnik 2018, 20).
} 
dienten die Irritationen bzw., wertneutraler gefasst, auch Momente der Verwunderung und Überraschung in den Aussagen der Teilnehmerlnnen zu Kindheit in Deutschland. Auch die Momente der Irritation und Überraschung, die die beteiligten ForscherInnen im Verlauf der Datenerhebung erlebten, wurden in Interpretationssitzungen ausgetauscht und reflektiert, um die eigenen impliziten Erwartungen an die Teilnehmerlnnen zu rekonstruieren (Charmaz 2014) und damit auch die eigene Position im Forschungsprozess, bspw. hinsichtlich machtförmiger (generationaler/hegemonialer) Strukturen, zu reflektieren (Scharathow 2010, 91 f.).

Auf Grundlage von ersten, noch stark am Material orientierten Konzepten wurden axiale Kodierdurchgänge vorgenommen (Strauss/Corbin 1990), aus denen in Auseinandersetzung mit den Relationen zwischen den Konzepten abstraktere Kategorien entwickelt wurden. Herausgearbeitet wurden zwei Hauptkategorien für die Deutungs- und Erfahrungskomplexe (veranstaltete und sichere Kindheit) mit je zwei Unterkategorien und für die Strategien drei Kategorien (Nutzen der Schule, Zugang zu Kindheitsräumen, Draußensein kultivieren).

\section{ERGEBNISSE DER ANALYSE}

Im Folgenden werden anhand von Beispielmaterial aus den Gruppendiskussionen die Deutungsund Erfahrungskomplexe sowie die Strategien neu zugewanderter Kinder im Umgang mit Kindheit in Deutschland präsentiert. ${ }^{9}$ Im Verlauf der Analyse wurden zwei Hauptkategorien für die Deutungs- und Erfahrungskomplexe gebildet: 1. Die „veranstaltete Kindheit" mit den Unterkategorien a) raum-zeitliche Separierung und b) Kindzentriertheit der Familie und 2. die (relativ) "sichere Kindheit" mit den Unterkategorien a) Schule/institutioneller Raum und b) öffentlicher Raum. Das zweite Unterkapitel präsentiert drei Strategien, die die Befragten auf Grundlage dieser beiden Deutungs- und Erfahrungskomplexe, also auf Grundlage bisheriger Erfahrungen und neuer Situationsdeutungen, entwickeln: das Nutzen von Schule, die Suche nach Zugängen zu
Kindheitsräumen und das Kultivieren des DrauBenseins.

\subsection{Deutungs- und Erfahrungskomplexe}

Die Deutungs- und Erfahrungskomplexe wurden in erster Linie aus den Schilderungen der TeilnehmerInnen rekonstruiert, in denen diese Irritation und Verwunderung über bestimmte Aspekte von Kindheit in Deutschland schilderten, und die sie mit Erfahrungen aus den Herkunftsländern verglichen bzw. kontrastierten.

\subsubsection{Kindheit in Deutschland als veranstaltete} Kindheit

"Also ich komme aus dem Kosovo. Und es ist komplett anders als im Kosovo. Weil, also im Kosovo, wir haben nur halbtags Unterricht. Und hier ist so mehr in der Schule. Aber wir im Kosovo verbringen mehr Zeit mit Freundin. Wir gehen zusammen miteinander und machen so mehr Spaß. Hier ist so Freizeit gehen Kurs, also Tanzkurs oder Ballspielen. Und es ist komplett anders, wenn zum Beispiel ersten Monat, hier, also hier für mich war es sehr schwierig." (Naime, 13)

Das Zitat von Naime repräsentiert die raum-zeitliche Separierung als erste Unterkategorie der veranstalteten Kindheit. Sie erlebt diese als räumlich und zeitlich straffer strukturiert, als sie es aus ihrem Herkunftsland gewohnt ist. Naime beginnt mit einem Verweis auf die Schule im Kosovo, die nur halbtags stattfinde, wodurch mehr Zeit mit den Freundinnen bleibe. Diese wird als unkomplizierte, freudvolle gemeinsame Zeit skizziert - und von Naime in einen Kontrast zur Freizeit hier gestellt. Hier gehe man in einen Kurs, bspw. in einen Tanz- oder Ballspielkurs. Naime erlebt diese Art der Freizeitgestaltung als komplett anders, was für sie zu Beginn sehr schwierig gewesen sei. Kindheit in Deutschland findet aus ihrer Perspektive in der Schule und, nach der Schule, in von Erwachsenen veranstalteten Kursen statt. Sie zeichnet sich damit durch eine raum-zeitliche Taktung aus, die voraussetzungsvoll ist, und deren komplexe Zugangsregeln von den Neuankömmlingen erst erschlossen werden

\footnotetext{
${ }^{9}$ Das in diesem Kapitel präsentierte Material wurde vollständig anonymisiert und zum Zweck einer leichteren Lesbarkeit geringfügig geglättet. Die Abkürzung I steht für die Interviewerin. Kursivsetzungen im Fließtext dienen der Kennzeichnung von Formulierungen, die direkt aus dem Material übernommen wurden.
} 
müssen. Die Routine, die Naime im Kosovo entwickelt hatte, nämlich, sich nach der Schule einfach zu treffen und miteinander Zeit zu verbringen, ist hier nicht mehr möglich. Die bekannten Wege, Peerkontakte zu pflegen und (neue) Freundschaften zu knüpfen, führen nicht zum Ziel. Ähnlich geht es Samir. Er erzählt von seiner ersten Zeit in Deutschland:

„Und mir war irgendwie langweilig. Keine Ahnung. Man kannte einfach nicht die Sprache. Man hatte keine Freunde. Alle Freunde waren in anderem Land. Dann einfach über Skype geredet mit Freunden. War so bei mir. Und ich habe auch manchmal so Spiele gespielt mit ihnen, die so online gehen. Und dann habe ich mich irgendwie gewöhnt. Meine Mutter hat gesagt, du bist irgendwie geworden wie deutsche Kinder. Weil die sind die ganze Zeit auf dem Handy. Aber eigentlich war ich nicht so. Weil in Serbien war ich eigentlich immer draußen. In Serbien ist das so, egal wie viel Uhr, immer ist einer draußen, ein Kind. Immer." (Samir, 11)

Aus der Sequenz geht hervor, dass das DrauBensein für Samir in Serbien garantierte, andere Kinder zu treffen. Die Möglichkeit, zu jeder Uhrzeit nach draußen gehen und ohne weiteren (Organisations-)Aufwand andere Kinder treffen zu können, greift für inn gegenwärtig nicht mehr. Samir schildert, dass es zunächst die (noch nicht beherrschte deutsche) Sprache gewesen sei, die inn dazu brachte, vor allem mit den Freunden im anderen Land zu reden und mit ihnen online zu spielen. Er habe sich dann daran gewöhnt, was seine Mutter zu der Bemerkung führt, er sei geworden wie deutsche Kinder [...] die ganze Zeit auf dem Handy. Samirs Darstellung lässt vermuten, dass es nicht in erster Linie Sprachschwierigkeiten sind, die die Etablierung von Freundschaften verhindern. Zwar stellen diese zunächst eine Hürde dar, entscheidend ist aber letztlich die andere Strukturiertheit von Kindheit in Deutschland. Seine Bemerkung, ich war eigentlich nicht so, kann dahingehend interpretiert werden, dass er sich (notgedrungen) an die Struktur der inn umgebenden, vor allem im privaten Raum stattfindenden, Kindheit angepasst hat. Muzafer, ein weiterer Teilnehmer an dieser Gruppendiskussion, äußert es als expliziten Wunsch, es möge sich mehr Leben draußen abspielen:
„Keine Ahnung. Dass mehr Leute draußen sind und nicht so drinnen sind. Die ganze Zeit so. Klar, wenn es regnet. Klar, verstehe ich das. Aber wenn es nicht regnet, sind auch fast keine Leute draußen." (Muzafer, 10)

Auch aus dem Zitat von Muzafer geht deutlich seine Irritation hinsichtlich der Absenz von Leuten (insbesondere Kindern) im öffentlichen Raum hervor. Auch für inn ist draußen klar der Ort, an dem Peerinteraktionen stattfinden. Muzafer rätselt, warum die Leute vor allem drinnen seien, selbst wenn es nicht regnen würde.

Mit der Kindzentriertheit der Familie wird die zweite Unterkategorie der veranstalteten Kindheit erfasst, die bei den Teilnehmerlnnen der Gruppendiskussionen Verwunderung hervorruft und zu (Neu-)Deutungen der Situation anregt. Im Vordergrund steht der immense Aufwand, den Erwachsene, vor allem die Mütter, für ihre Kinder betreiben. Dafür steht die folgende Sequenz beispielhaft:

„R: Ich finde, dass es irgendwie bisschen anderes. Weil ich war bei einem Mädchen. Sie hat Geburtstag gehabt, also sie hat eine Übernachtungsparty gemacht. Und wir, also da haben, hat Mama alles gemacht. Zum Beispiel: Ich weiß nicht, wie war das nur so an der Party oder so? Aber ich finde, also die hat das alles, sie hat nur, sie hat gekocht, sie hat so sauber gemacht. Also sie hat so nachts, ich weiß nicht wie das heißt [...].

I: Ach die Betten, Bettwäsche, Bettdecken und so?

R: Ja, das hat sie alles so schön gemacht. Und so dann morgens, wir haben so einen Film geguckt, gegessen und so. Sie hat so uns, ja, ich habe mich so gefreut. Weil also...

I: Sie hat alles für euch gemacht?

R: Ja. Meine Mutter würde es auch machen, aber nicht so. Aber natürlich nicht immer, weil sie arbeitet auch. Und also sie ist nicht da, eigentlich immer. Und deswegen ja, wir haben auch ein Fotoshooting gemacht, ja." (Rita, 13) 
Rita schildert die Geburtstagsfeier eines Mädchens aus ihrer Schulklasse, um zu erläutern, warum sie Kindheit in Deutschland irgendwie ein bisschen anders findet. Den Aufwand, mit dem die Mutter der Klassenkameradin die Übernachtungsparty für die Tochter und ihre Gäste vorbereitet, kommentiert Rita verwundert. Sie fragt sich, ob dies nur so an der Party liege oder - so kann man ihren Gedanken ergänzen - ob die Mutter auch ansonsten alles machen würde. Rita schildert die vielen Leistungen der Mutter: Kochen, Saubermachen, die Betten herrichten, einen Film und ein Fotoshooting für den nächsten Tag vorbereiten. Rita äußert ihre Freude, aber auch ihre Irritation über die viele Mühe der Mutter, für die Mädchen alles so schön gemacht zu haben. Sie zieht den Vergleich zu ihrer eigenen Mutter, die es zwar auch so machen würde, aber eben doch nicht ganz so hingebungsvoll bzw. unter so großem zeitlichem Aufwand - was Rita damit erklärt, dass ihre Mutter berufstätig und dadurch nicht so viel zu Hause sei. Die von ihr beobachteten Praktiken eines „intensiven parenting" (Faircloth 2014) und des immensen Aufwands, der hier von der Mutter betrieben wird, entsprechen offenbar nicht Ritas bisherigen Erfahrungen. Deutlich wird, wie sie an der Interpretation dieser Situation arbeitet, bspw. indem sie die Hypothese aufstellt, es handle sich um eine Ausnahme, die das besondere Ereignis der Geburtstagsfeier beträfe. Ritas Irritation bezüglich der Kindzentriertheit der Familie, die ein zentrales Merkmal des normativen Musters „guter Kindheit" in Deutschland darstellt (Bühler-Niederberger 2020a, 34 ff.), wird am präsentierten Beispiel eines außergewöhnlichen Ereignisses wie der geschilderten Geburtstagsfeier besonders deutlich. Aber auch hinsichtlich alltäglicherer Praktiken heben die Befragten die Unterstützungsleistungen der Eltern einheimischer Kinder hervor. Angesichts der raum-zeitlich separierten und ins Private verlagerten Kindheit stellen diese Unterstützungsleistungen tatsächlich auch eine Notwendigkeit dar: Bereitschaft und Ressourcen der Eltern sind eine Voraussetzung, damit diese Art der Kindheit überhaupt stattfinden kann - um an besagten Kursen teilnehmen zu können oder im Privaten einen Raum zu schaffen, um die Freundlnnen dorthin einladen zu können. Die Irritation der neu zugewanderten Kinder über die „veranstaltete Kindheit" bezieht sich auf eine Kindheit, die in
Deutschland an den Vorstellungen und Ressourcen der Mittelschicht orientiert ist.

\subsubsection{Kindheit in Deutschland als (relativ) sichere Kindheit}

Die erste Unterkategorie des Deutungs- und Erfahrungskomplexes von Kindheit in Deutschland als sichere Kindheit bezieht sich auf den institutionellen Raum der Schule als zentralen Kindheitsort. Die aktuell von ihnen besuchten Schulen beschreiben die Teilnehmerlnnen der Gruppendiskussionen als sichere Orte, zumindest hinsichtlich ihrer offiziellen Vertreterlnnen, der Lehrkräfte. Diese werden von den Befragten als unterstützend und vertrauenswürdig beschrieben. Es sei ihren Lehrerlnnen wichtig, dass sie etwas lernten, aber auch, dass sie Spaß an der Schule hätten. Sie werden als Ansprechpersonen wahrgenommen, die bei Problemen hinzugezogen werden können. Die Deutungen von Schule als ein sicherer Ort und von Lehrerlnnen als unterstützende Personen werden in den Gruppendiskussionen, insbesondere durch die teilnehmenden Jungen, mit den Erfahrungen aus den Herkunftsländern kontrastiert, in denen Schule als Ort der Gewalt gegen Kinder erscheint:

„Wir haben in unserer Klasse viele Kinder aus Marokko. Und die haben das viel erzählt. Aber so ist das noch schwer. Wegen Krieg, Schule immer geschlagen worden. Die bekommen ja immer auf die Hände Schläge. Wenn wir zehn bekommen, ist das gut. Wenn wir nur einen Fehler haben, bekommen wir Schläge. Aber in Deutschland ist das anders." (Sinan, 11)

Sinan gehört selbst nicht zu den Kindern aus Marokko; gleichwohl verknüpft er deren Schilderungen von Bestrafungen in der Schule mit seinen Erfahrungen, die er in der Schule in Syrien gemacht hat. Und Mahir ergänzt:

„In Ägypten man darf auch Kinder schlagen. [...] Da habe ich schon mal so Krankenwagen gesehen, weil eine Lehrer hat da ein Kind geschlagen, weil dings, weil der seine Hausaufgaben nicht gemacht hat. Der Junge war am Bluten. Der hat ihn mit so einem Stock geschlagen." (Mahir, 12) 
A: (Zum Beispiel deine Haare sind schwarz.)

E: Ja, in meiner alten Klasse waren alle Deutsche. Und sie sagten mir so und Schwarzkopf ist so wie eine Beleidigung." (Eliya, 10/Mahir, 12/ Adam, 11)

„M: Also da waren so Jugendliche. Dann haben die uns gesagt: ,Habt ihr deutsches Pass?' Wir haben ,nein' gesagt. Also ich und [unverständlich] gestern und dann kommen die, streiten. ,Ausländer, ich hasse Ausländer.'“ (Murad, 11)

ben gesagt, als wir noch [in] Syrien waren, wenn jemand euch anspricht, dann dürfen wir mit keinen Leuten reden, weil die sagen einfach: ,Kannst du mir helfen?', zum Beispiel ein alter Mann. Denn keiner sagt zu einem alten Mann nein. Dann kommst du mit und dann nehmen die dich einfach in ein Auto rein." (Murad, 11)

Weitere Erfahrungen von Unsicherheit in den Herkunftsländern betreffen öffentliche Strukturen wie bspw. die Polizei oder den Rettungsdienst. Weder auf Polizistlnnen noch auf Rettungshelferlnnen könne man sich verlassen; sie kämen entweder gar nicht oder viel zu spät und würden dann womöglich nicht einmal helfen. Gemessen an diesem Maßstab erscheint die Kindheit in Deutschland als sicher, öffentliche Strukturen als verlässlich, Lehrerlnnen als unterstützend. Dem Gewaltverbot gegen Kinder, aber auch gegen andere Gruppen, kommt in den Augen der Befragten Gültigkeit zu und der Schutz der eigenen Person kann erwartet werden. Diese Interpretation vom öffentlichen (und institutionellen) Raum in Deutschland als einen sicheren Raum für Kinder hält vor allem im Vergleich zu den Erfahrungen aus den Herkunftsländern stand. Denn auch in Deutschland ist der öffentliche Raum nicht bar von Gefahren. Aus den Gruppendiskussionen können vielmehr neue Gefahren oder Bedrohungen rekonstruiert werden, die vor allem den Status der Befragten als neu Zugewanderte betrifft. Die Teilnehmerlnnen berichten von Erlebnissen der Ausgrenzung und rassistischer Anfeindungen:

„E: Und alle kennen dieses Wort Schwarzkopf. Leute, die Schwarzkopf sind, sind Ausländer.

M: (Was ist Schwarzkopf?)

E: Die sind nicht gut.
Anhand der Darstellungen der Teilnehmerlnnen kann der öffentliche Raum und mitunter auch die Peerinteraktionen im schulischen Kontext als ein potenziell bedrohlicher Raum rekonstruiert werden. Der Deutungs- und Erfahrungskomplex von Kindheit in Deutschland als eine sichere Kindheit muss dahingehend differenziert werden, dass die Befragten Deutschland als sicher erleben - in Hinblick auf ihren Status als Kind, dem Schutz und Unterstützung zustehen. In ihrer Zugehörigkeit bzw. Zuschreibung zur Gruppe der Leute aus anderen Ländern machen sie hingegen (neue) Erfahrungen der Unsicherheit und des Bedrohtseins. Wenn Deutschland ihnen auch Sicherheit als Kinder bietet, so mutet es ihnen als neu Ankommende auch neue Unsicherheiten zu.

Mit den Deutungs- und Erfahrungskomplexen wurden die Deutungen von Kindheit in Deutschland in Abgrenzung zu den Erfahrungen neu zugewanderter Kinder aus den Herkunftsländern rekonstruiert. Im Folgenden sollen nun die Strategien dargestellt werden, die die Befragten auf Grundlage dieser Deutungen entwickeln, um an der „veranstalteten“ und (relativ) „sicheren“ Kindheit in Deutschland teilzunehmen und sich in der neuen Lebenswelt zu positionieren. Alle drei Strategien sind darauf ausgerichtet, Möglichkeiten der Peerinteraktionen zu schaffen oder zu erweitern, indem sie 1. die Schule nutzen, 2. sich Zugang zu (separierten) Kindheitsräumen verschaffen und 3. das Draußensein kultivieren.

\subsubsection{Die Schule nutzen}

Als verpflichtender Ort der Kindheit jenseits der separierten Kindheitsräume bietet die Schule die

\subsection{Strategien des Umgangs}


Möglichkeit, Kontakte mit Peers zu knüpfen und Freundschaften aufzubauen. Diese Möglichkeit nutzen die befragten Kinder maximal - mit Erfolg; die Gruppendiskussionen enthalten viele Hinweise, dass die Kinder ihre (oft zahlreichen) Freundlnnen vor allem aus der Schule kennen. ${ }^{10}$ Dabei sind es nicht nur die Unterrichts- und Pausenzeiten, in denen die Interaktionen stattfinden, sondern auch die Schulwege und die Nachmittagsangebote wie der betreute Ganztag oder Sport-AGs. Das Nutzen von Schule für die Interaktion mit Peers kann als eine Strategie des Umgangs mit der veranstalteten Kindheit interpretiert werden, die zu einem großen Teil in spezifischen Räumen bzw. im privaten Raum stattfindet, zu denen der Zugang voraussetzungsvoll ist. Aber auch der zweite Deutungsund Erfahrungskomplex - das Erleben von Schule als sicheren Raum - trägt dazu bei, dass die Schule wertgeschätzt und sowohl für die peerbezogenen Anliegen als auch für Bildungsziele im engeren Sinne genutzt wird. Die Lehrkräfte und die Freundlnnen werden als UnterstützerInnen angesprochen:

„S: Als ich in der Schule kam ersten Tag hat er mich geholfen, die Schule kennenzulernen. Dann ist er mein Freund. Dann ich kannte keine Kinder. Die haben mich geschlagen. Dann hat er mir auch geholfen.

I: Wie hat er dir geholfen? [...]

S: Er hat drei Lehrer geholt." (Samir, 11)

Die befragten Kinder machen Schule außerdem hinsichtlich ihrer Bildungs- und Zukunftsvorstellungen zu einem zentralen Ort, indem sie ein Maximum an Mühe in die Bewältigung schulischer Anforderungen legen, um rasch die deutsche Sprache zu lernen, in die nächste Klasse versetzt zu werden und möglichst eine gute weiterführende Schule besuchen zu können. Die Teilnehmerlnnen der Gruppendiskussionen sind sich einig darüber, dass auch die Eltern der Schule bzw. dem schulischen Erfolg und Engagement der Kinder eine zentrale Rolle zuweisen:
„Ja bei mir ist es auch so wie bei Pipa. Und ichich wünsche nur, dass meine Eltern einen Tag verstehen, dass ich so viel lerne. Weil die Klasse wiederholen, es ist für mich nicht gut natürlich, deshalb will ich immer lernen und immer so die Beste sein, also die Erste sein. Und dass sie verstehen, dass ich ALLEINE so will lernen und sie es mir nicht immer sagen müssen." (Edita, 12)

Ihren Wunsch nach schulischem Erfolg und sozialem Aufstieg verknüpft Edita mit der Schuldigkeit gegenüber dem Vater, der viele Jahre allein in Deutschland gelebt habe, um die Migration der Familie zu ermöglichen:

„Also und dann ich habe nicht so mit meinem Papa gelebt. Weil der war hier in Deutschland. Und also er hat alles so für uns gemacht, dass wir, also kommen hier in Deutschland, lernen, also einen gutes Beruf haben, eine gute Zukunft sozusagen. Er hat so soviel für uns gemacht. Also damit wir Schule haben und so." (Edita, 12)

Die Zitate weisen darauf hin, dass der Ehrgeiz zu lernen und in der Schule erfolgreich zu sein auch in Belastung umschlagen kann, bspw. wenn die Eltern zu hohen Druck aufbauen und keine Zeit mehr für anderes bleibt. Die umfassende Bedeutung von Schule - als Bildungsort und als Ort der Peerinteraktion für neu zugewanderte Kinder verweist darauf, wie sehr sie auf die konkrete von innen besuchte Schule, deren (Nachmittags-)Angebote, Gestaltung des Unterrichts und Integrationskonzepte angewiesen sind. Die "Scholarisierung" von Kindheit in Deutschland, verstanden als zeitliche Ausdehnung der Schule, aber auch der wachsenden Bedeutung, die der Schule im Leben von Schülerlnnen und ihren Familien zukommt (Fraij et al. 2015), erfährt bei neu zugewanderten Kindern nochmals eine zusätzliche Betonung.

\subsubsection{Zugänge zu den separierten Kindheitsräu- men schaffen}

Aus dem Material konnte eine weitere Strategie der Teilnehmerlnnen rekonstruiert werden, um den segregierenden Effekten der veranstalteten

\footnotetext{
10 Dies bestätigte sich auch in den individuellen Interviews, in denen die Kinder Netzwerkkarten über familiale und freundschaftliche Kontakte erstellten.
} 
Kindheit zu begegnen. Sie besteht darin, sich Zugänge zu den separierten und privaten Räumen zu verschaffen. Die Kinder berichten bspw. davon, Freundlnnen, die sie in der Schule kennengelernt hatten, zunächst zum Training im Verein begleitet und sich schließlich ebenfalls dort angemeldet zu haben. Oder sie erwähnen Vereine und ähnliche Kontexte wie bspw. Tanzschulen, wo sie einen Kurs besuchen. Auch niedrigschwellige (weniger strikt generational separierte, aber dennoch kindspezifische) Orte wie Freibäder, Zoos oder Kinos werden als Orte benannt, an denen Freizeit verbracht wird.

Teil dieser zweiten Strategie ist es auch, den privaten Raum - der eigenen Familie oder denjenigen der Freunde - für die Peerinteraktionen zu etablieren, indem bspw. eine explizitere Verabredungskultur entwickelt und gepflegt wird. In vielen Fällen wird es nötig, die Eltern stärker als zuvor in die Pflicht zu nehmen. Die Kinder schildern, wie sie ihre Mütter instruieren, Kontakt zu den Mitschülerlnnen bzw. zu deren Müttern aufzunehmen. Auf diese Weise hoffen sie, Bekanntheit und Vertrautheit zu schaffen, sodass die Kinder einander besuchen dürfen. Eine Teilnehmerin berichtet, wie sie und ihre Schwester jeweils ihre Freundinnen zu einer Übernachtungsparty nach Hause einluden, damit die Eltern diese kennenlernen und weiteren gemeinsamen Unternehmungen zustimmen würden:

„Also Beispiel meine Familie, wenn sie meine besten Freunde die Familie nicht kennen, dann vielleicht kann ich nicht mit ihnen rausgehen. Pipas Freundinnen aus ihrer Klasse, sie haben einmal bei uns übernachtet und so. Sie haben die Eltern kennengelernt und so, eigentlich muss man Kontakt und so haben." (Sadaf, 15)

\subsubsection{Das Draußensein kultivieren}

Die dritte Strategie kann gewissermaßen als komplementär zur zweiten betrachtet werden. Während es bei letzterer um das Erschließen von Zugängen zu den segregierten und privaten
Kindheitsorten, im Sinne eines Einfügens in die veranstaltete Kindheit, geht, umfasst die dritte Strategie Bemühungen, das Draußensein für sich selbst bzw. für Peerinteraktionen zu kultivieren. In den Gruppendiskussionen werden geplante oder bereits realisierte Initiativen thematisiert, sich den öffentlichen Raum, oft mit der Unterstützung von Freundlnnen, Geschwistern und Cousins, zu erschließen. Dazu organisieren sie niedrigschwellige, aber legitime Unternehmungen wie bspw. den Besuch von Fastfood-Restaurants und das Spazierengehen im Park oder in der Stadt. Damit knüpfen sie an Erfahrungen der Sozialität an, die nicht auf Kinderorte abzielt, sondern auf (weniger generational segregierte) Alltagsorte. Den Fastfood-Restaurants (wie türkische oder asiatische Imbisse, McDonald's oder Eisdielen) scheint dabei eine wichtige Bedeutung zuzukommen. Wie nur wenige andere öffentliche bzw. frei zugängliche Orte bieten sie eine niedrigschwellige Möglichkeit des geselligen Zusammenseins. Außerhalb der stärker veranstalteten (und pädagogisierten) Orte (wie bspw. auch Vereinen, Jugendzentren etc.) stehen Kindern nur wenige Räume für diesen Zweck zur Verfügung. Die beiden oben herausgearbeiteten Deutungs- und Erfahrungskomplexe spielen auch hier zusammen: Die Erfahrung aus dem Herkunftsland, dass das Draußensein eine legitime Art des Kindseins ist, und die Wahrnehmung vom öffentlichen Raum als ein (relativ) sicherer Raum ermöglichen die Entwicklung dieser Strategie.

\section{ZUSAMMENFÜHRENDE DISKUSSION DER ERGEBNISSE UND RESÜMEE}

Das zentrale Interesse des vorliegenden Beitrags gilt dem Blick neu zugewanderter Kinder auf Kindheit in Deutschland und ihren Umgangsweisen mit dieser Kindheit. Ausgangspunkt der Analyse waren die Irritationen, die die Kinder in Hinblick auf Kindheit in Deutschland erleben, und die Erfahrungen aus den Herkunftsländern, die diese zur Kontrastierung der von ihnen erlebten Kindheiten heranziehen. ${ }^{11}$

\footnotetext{
${ }^{11}$ Der analytische Zugang legt den Fokus auf diese markanten Unterschiede zwischen dem Kindsein in den Herkunftsländern und in Deutschland. Weniger in den Blick geraten die von den Kindern erlebten Gemeinsamkeiten ihrer Kindheiten „dort“ und „hier“, etwaige ähnliche Erfahrungen oder gleichbleibende Deutungsmuster. Damit ist eine klare Begrenzung der Aussagekraft der Studie benannt, die auf dem hier gewählten methodologischen Zugang beruht.
} 
Herausgearbeitet wurden zunächst zwei Deutungs- und Erfahrungskomplexe. Zum einen derjenige der "veranstalteten Kindheit“, welcher darauf verweist, dass Kindheit in Deutschland im Kontrast zu den Herkunftsgesellschaften als stärker separiert und als raum-zeitlich stark getaktet erlebt wird. Die befragten Kinder haben nur begrenzte Zugänge zu diesen Räumen, die vorrangig im privaten Bereich der Familien liegen oder über diese organisiert werden. Rekonstruiert wurde außerdem die Irritation über die ausgeprägte Kindzentriertheit der Familien, die in den Gruppendiskussionen thematisiert wurde. Dieser Blick neu zugewanderter Kinder entspricht Befunden, die auch von SozialwissenschaftlerInnen über die Struktur von Kindheit in Deutschland, ihre Ausgestaltung und ihre an den Vorstellungen und Möglichkeiten der Mittelschicht ausgerichteten normativen Muster vorgelegt wurden. Die hier vorgelegte Analyse zeigt darüber hinaus, dass es diese „normale“ Kindheit ist, die für die Gruppe neu zugewanderter Kinder Zumutungen (in Form eines hohen Aufwands, der für die Ermittlung der mitunter impliziten Regeln und Zugänge erbracht werden muss) bereit hält. Es handelt sich dabei um Hürden im Integrationsprozess, die spezifisch sind für Kinder. Gleichwohl - und das betrifft den zweiten Deutungs- und Erfahrungskomplex der "sicheren Kindheit" - erleben sie Kindheit in Deutschland als einen (öffentlichen) Raum, der sie als Kinder schützt und innen Gewaltfreiheit zusichert. Insofern erleben die Befragten ihre Position als Kinder in Deutschland auch als einen Gewinn. Dies muss jedoch in Hinblick auf die Intersektion mit der Zugehörigkeit zur Gruppe neu zugewanderter Menschen relativiert werden. Ausgrenzungen und Diffamierungen - sowohl im schulischen als auch im öffentlichen Raum - wurden in den Gruppendiskussionen mehrfach zum Thema gemacht. Während die Position als Kind in Deutschland also einen Gewinn darstellt, so birgt der Status als Zugewanderte/r auch (neue) Bedrohungen. An die in Kapitel 2.2 (holzschnittartig) skizzierten Charakteristika von Kindheit im Globalen Süden lassen sich die Befunde insofern anschließen, als dass die Befragten ihre Kindheit "dort" als weniger vor Gewalt und Gefahren geschützt erlebten; dies gilt für den öffentlichen aber insbesondere auch für den schulischen Raum. In den Schilderungen der Erfahrungen er- scheint Kindheit in den Herkunftsländern außerdem weniger separiert und ergo stärker integriert in das gemeinsame soziale Leben der Altersgruppen. Dies kann in Verbindung gesetzt werden zu den stärker kollektiv bzw. interdependent ausgerichteten Orientierungen, die sich durch eine (frühe) Funktionalität für die Familie auszeichnen und Kindheit (und Jugend) ggf. weniger als Schonraum und Moratorium ausgestalten. Vor diesem Hintergrund kann die hohe schulische Erfolgs- und Aufstiegsorientierung der hier zu Wort kommenden Kinder (auch) als eine Verpflichtung gegenüber der Familie interpretiert werden. Für eine differenziertere Analyse der Erfahrungen in den Herkunftsländern und ihre Implikationen für die Deutungen von Kindheit in Deutschland wäre letztlich eine methodische Anlage nötig, die die Biographien der Kinder expliziter in den Blick nimmt, als es hier der Fall war.

Der Blick auf die Strategien der befragten Kinder im Umgang mit Kindheit in Deutschland verweist auf ihre kreative und kompetente Akteurlnnenschaft. Die drei in der vorliegenden Analyse rekonstruierten Strategien beziehen sich in erster Linie auf das Anliegen der Kinder, die Zugangshürden für Interaktionen mit den Peers, d. h. die Separiertheit von Kindheit in Deutschland, zu bewältigen. Die erste Strategie des Nutzens der Schule kann als pragmatische Strategie interpretiert werden, ohne großen zusätzlichen Aufwand mit den Peers zu interagieren und Freundschaften aufzubauen. Mit der zweiten Strategie werden wiederum Bemühungen deutlich, Zugang zu den separierten Orten der Kindheit zu erhalten, sowohl was organisierte Freizeitaktivitäten betrifft als auch das Einbringen in eine Verabredungskultur mit den Peers. Diese Strategie kann als „kompetente Gefügigkeit“ (Bühler-Niederberger 2020a, 238) hinsichtlich der vorherrschenden generationalen Strukturen gelesen werden: Indem Kinder sich auf die raum-zeitliche Taktung bspw. von kursförmigen Angeboten einlassen oder die eigenen Eltern zur Mitorganisation von Verabredungen über die Eltern der anderen Kinder heranziehen, verfolgen sie eine aktive Einpassung in die separierte Kindheit. Die dritte Strategie, das Draußensein zu kultivieren, beruht wiederum auf Erfahrungen aus dem Herkunftsland, wo der öffentliche Raum bzw. die Nachbarschaft als weniger veranstalteter und dafür leicht zugänglicher Ort für Peerinteraktion genutzt 
wurde. Die Aneignung von weniger kindspezifischen bzw. weniger von Erwachsenen kontrollierten Räumen kann damit als Strategie interpretiert werden, den begrenzten Handlungsraum des Kindseins in Deutschland zu erweitern. Bedingung für diese Strategie des Draußenseins ist wiederum die Deutung von Kindheit in Deutschland als eine sichere Kindheit. Aus den drei Strategien geht das breite Spektrum aktiver Bemühungen hervor, die neu zugewanderte Kinder angesichts der herausfordernden Kindheit in Deutschland erbringen.

Dabei soll nicht aus dem Blick geraten, dass die kompetente Bearbeitung der eigenen Situation nicht in allen Fällen gelingt, denn diese ist nicht nur von den Deutungs- und Interpretationsleistungen der Kinder abhängig, sondern auch von einer Vielzahl situativer und struktureller Faktoren, die außerhalb ihrer Gestaltungsmöglichkeiten liegen. Generationale Ordnungsstrukturen, die die Handlungsräume von Kindern stark begrenzen, fallen zusammen mit der Zugehörigkeit zur Gruppe der neu Zugewanderten: Letztere geht mit restriktiven rechtlichen Begrenzungen bzw. strukturellen Benachteiligungen einher, wie sie eingangs skizziert wurden. Diese strukturellen Benachteiligungen limitieren auch den Erfolg der von den Kindern unternommenen Bemühungen, mit den Eigenarten von Kindheit in Deutschland - insbesondere deren Separiertheit und Privatheit - umzugehen. Isolation und Einsamkeit, andauernde Langeweile oder der Rückzug in die Familie können die Folge sein, wenn die gewählten Strategien dauerhaft erfolglos bleiben. Gleichwohl eröffnet die (relative) Sicherheit der Schule und des öffentlichen Raumes auch Gelegenheiten, sich Räume der (Peer-)Interaktion anzueignen und die individuellen schulischen und außerschulischen Anliegen zu verfolgen.

\section{LITERATUR}

Agar, Michael (2006): An Ethnography By Any Other Name. Forum Qualitative Sozialforschung, 7 (4), Art. 36.

Alanen, Leena (2005): Kindheit als generationales Konzept. In: Hengst, Heinz/Zeiher, Helga
(Hrsg.): Kindheit soziologisch. Wiesbaden: Springer VS, 65-82.

Bauer, Elaine (2016): Practising kinship care: Children as language brokers in migrant families. In: Childhood, 23 (1), 22-36.

Blumer, Herbert (1969): Symbolic Interactionism. Perspective and Method. Berkeley: University of California Press.

Bohn, Irina/Landes, Benjamin/Seddig, $\mathrm{Na-}$ dine/Warkentin, Stephanie (2016): „Ich brauche hier nur einen Weg, den ich finden kann“. Ankommen und Einleben in NRW aus der Sicht geflüchteter Kinder und Jugendlicher. Online verfügbar unter: https://www.iss-ffm.de/fileadmin/assets/veroeffentlichungen/downloads/ 641_yr-zwischenbericht_web_neuesimpressum_einzeln.pdf (20.03.2021).

Bohnsack, Ralf (2003): Differenzerfahrungen in der Identität und des Habitus. Eine empirische Untersuchung auf Basis der dokumentarischen Methode. In: Liebsch, Burkhard/Straub, Jürgen (Hrsg.): Lebensformen im Widerstreit. Integrations- und Identitätskonflikte in pluralen Gesellschaften. Frankfurt am Main: Campus, 136-160.

Bohnsack, Ralf (2012): Orientierungsschemata, Orientierungsrahmen und Habitus. In: Schnittenhelm, Karin (Hrsg.): Qualitative Bildungs- und Arbeitsmarktforschung. Grundlagen, Perspektiven, Methoden. Wiesbaden: Springer VS, 119-153.

Bourdillon, Michael/Boyden, Jo (2014): Growing Up in Poverty. Findings from Young Lives. London: Palgrave MacMillan.

Brüggemann, Christian/Nikolai, Rita (2016): Das Comeback einer Organisationsform: Vorbereitungsklassen für neu zugewanderte Kinder und Jugendliche. Friedrich-Ebert-Stiftung. Online verfügbar unter: https://library.fes.de/pdf-files/studienfoerderung/12406.pdf (20.03.2021).

Bühler-Niederberger, Doris (2020a): Lebensphase Kindheit. 2. überarbeitete Auflage. Weinheim: Beltz Juventa.

Bühler-Niederberger, Doris (2020b): Generationale Verpflichtungen - normative Muster und ihre Umsetzung. In: Berliner Journal für Soziologie, 30, 49-78.

Bühler-Niederberger, Doris/Schwittek, Jessica (2014): Young children in Kyrgyzstan: Agency in 
tight hierarchical structures. In: Childhood, 21 (4), 502-516.

Charmaz, Kathy (2014): Constructing Grounded Theory. Thousand Oaks: Sage Publications.

Crafter, Sarah/Cline, Tony/de Abreu, Guida/O'Dell, Lindsay (2017): Young peoples' reflections on what teachers think about family obligations that conflict with school: A focus on the non-normative roles of young caring and language brokering. In: Childhood, 24 (4), 517-530.

Dados, Nour/Connell, Raewyn (2012): The Global South. In: Contexts, 11 (1), 12-13.

Diekmann, Daniel/Fereidooni, Karim (2019): Diskriminierungs- und Rassismuserfahrungen geflüchteter Menschen in Deutschland: Ein Forschungsüberblick. In: Z'Flucht. Zeitschrift für Flucht- und Flüchtlingsforschung, 3 (2), 343360.

Eisenhuth, Franziska (2015): Strukturelle Diskriminierung von Kindern mit unsicherem Aufenthaltsstatus. Subjekte der Gerechtigkeit zwischen Fremd- und Selbstpositionierungen. Wiesbaden: Springer VS.

El-Mafaalani, Aladin (2013): Erziehungs- und Erwartungsdifferenzen im Alltag von Migrantenkindern. In: Zeitschrift für Grundschulforschung, 6 (1), 114-128.

El-Mafaalani Aladin/Kemper, Thomas (2017): Bildungsteilhabe geflüchteter Kinder und Jugendlicher im regionalen Vergleich. In: Zeitschrift für Flüchtlingsforschung, 1, 173-217.

Emmerich, Marcus/Hormel, Ulrike/Jording, Judith (2017): Prekarisierte Teilhabe. Fluchtmigration und kommunale Schulsysteme. In: Die Deutsche Schule, 109, 209-222.

Faircloth, Charlotte (2014): Intensive Parenting and the Expansion of Parenting. In: Lee, Ellie/Bristow, Jennie/Faircloth, Charlotte/Macvarish, Jan: Parenting Culture Studies. London: Palgrave MacMillan, 25-50.

Fraij, Amina/Maschke, Sabine/Stecher, Ludwig (2015): Die Scholarisierung der Jugendphase ein Zeitvergleich. In: Diskurs Kindheits- und Jugendforschung, 10 (2), 167-182.

Gerarts Katharina/Andresen, Sabine (2018): Erfahrungen auf der Flucht und die Bedeutung der
Flucht für Kinder und Familien. In: Hartwig, Luise/Mennen, Gerald/Schrapper, Christian (Hrsg.): Handbuch Soziale Arbeit mit geflüchteten Kindern und Familien. Weinheim: Beltz/Juventa, 162-173.

Goffman, Erving (2016): Stigma. Über Techniken der Bewältigung beschädigter Identität. 23. Auflage. Frankfurt am Main: Suhrkamp.

Günther, Marga/Wischmann, Anke/Zölch, Janina (2010): Chancen und Risiken im Kontext von Migration und Adoleszenz. Eine Fallstudie. In: Diskurs Kindheits- und Jugendforschung, 5 (1), 21-32.

Gurski, Lara/Rother, Timo (2018): Wie erleben geflüchtete Jugendliche Schule in verschiedenen Lerngruppen? In: Große Prues, Peter (Hrsg.): Inklusion im Rahmen von Schule - eine Aufgabe, viele Möglichkeiten. Osnabrück: Eigendruck, 173-184.

Heinzel, Friederike (2000): Methoden und Zugänge der Kindheitsforschung im Überblick. In: Heinzel, Friederike (Hrsg.): Methoden der Kindheitsforschung. Ein Überblick über Forschungszugänge zur kindlichen Perspektive. Weinheim: Juventa, 117-130.

Hofstede, Geert (1980): Culture's Consequences. Thousand Oaks: Sage.

Jaquemin, Melanie (2004): Children's domestic work in Abidjan, Côte d'Ivoire. The petites bonnes have the floor. In: Childhood, 11(3), 383397.

Kagitcibasi, Çiğdem (1996): Family and Human Development across Cultures. Hillsdale: Lawrence Erlbaum.

Kämpfe, Karin (2019): Kindheit in europäischen Migrationsgesellschaften. Orientierungen von Kindern im Kontext von Migration und Differenz. Wiesbaden: Springer VS.

Karakayali, Juliane (2018): (K)eine Frage der Wahl: Segregation und Grundschule in der Perspektive des institutionellen Rassismus. In: Migration und Soziale Arbeit, 2, 131-138.

Keller, Heidi (2007): Cultures of Infancy. New York: Lawrence Erlbaum.

Kitayama, Shinobu (2002): Cultural psychology of the self: a renewed look at independence and 
interdependence. In: von Hofsten, Claes/Backman, Lars (Hrsg.): Psychology at the Turn of the Millennium, Vol. 2: Social, Developmental and Clinical Perspectives. Hove, East Sussex: Psychology Press, 305-322.

Lechner, Claudia/Huber, Anna (2017): Ankommen nach der Flucht. Deutsches Jugendinstitut. Online verfügbar unter: https://www.dji.de/ fileadmin/user_upload/bibs2017/25854_lechner_huber_ankommen_nach_der_flucht.pdf (20.03.2021).

Massumi, Mona/von Dewitz, Nora (2015): Neu zugewanderte Kinder und Jugendliche im deutschen Schulsystem. Mercator-Institut für Sprachförderung und Deutsch als Zweitsprache und vom Zentrum für Lehrerlnnenbildung der Universität zu Köln. Online verfügbar unter: https://www.mercator-institut-sprachfoerderung.de/fileadmin/Redaktion/PDF/Publikationen/MI_ZfL_Studie_Zugewanderte_im_deutsch en_Schulsystem_final_screen.pdf (20.03.2021)

Mecheril, Paul (2002): Natio-kulturelle Mitgliedschaft - ein Begriff und die Methode seiner Generierung. In: Tertium Comparationis, 8, 104115.

Nohl, Arnd-Michael (2001): Migration und Differenzerfahrung. Junge Einheimische und Migranten im rekonstruktiven Milieuvergleich. Opladen: Leske und Budrich.

Oerter, Rolf (2013): Der Aufbau kultureller Identität im Spannungsfeld von Enkulturation und Akkulturation. In: Genkova, Petia/Ringeisen, Tobias/Leong, Frederick T. L. (Hrsg.): Handbuch Stress und Kultur. Interkulturelle und kulturvergleichende Perspektiven. Wiesbaden: Springer, 67-80.

Perkins, Jon Davis/Ajeeb, Maiss/Fadel, Lina/Saleh, Ghassan (2018): Mental health in Syrian children with a focus on post-traumatic stress: a cross-sectional study from Syrian schools. In: Soc Psychiatry Psychiatr Epidemiol, 53 (11), 1231-1239.

Qvortrup, Jens (2005): Kinder und Kindheit in der Sozialstruktur. In: Hengst, Heinz/Zeiher, Helga (Hrsg.): Kindheit soziologisch. Wiesbaden: Springer VS, 27-47.

Scharathow, Wiebke (2010): Vom Objekt zum Subjekt. Über erforderliche Reflexionen in der
Migrations- und Rassismusforschung. In: Broden, Anne/Mecheril, Paul (Hrsg.): Rassismus bildet: Bildungswissenschaftliche Beiträge zu Normalisierung und Subjektivierung in der Migrationsgesellschaft. Bielefeld: transcript, 87-111.

Soykoek, Seval/Mall, Volker/Nehring, Ina/ Henningsen, Peter/Aberl, Sigrid (2017): Post-traumatic stress disorder in Syrian children of a german refugee camp. In: The Lancet, 389 (10072), 903-904.

Strauss, Anselm/Corbin, Juliette (1990): Basics of Qualitative Research: Grounded Theory Procedures and Techniques. Thousand Oaks: Sage Publications.

Tangermann, Julian/Hoffmeyer-Zlotnik, Paula (2018): Unaccompanied Minors in Germany. Challenges and Measures after the Clarification of Residence Status. Working paper 80. Nürnberg: Federal Office for Migration and Refugees.

Thomas, William I. (1926): The Problem of Personality in the Urban Environment. In: Burgess, Ernest (Hrsg.): The Urban Community. Chicago: University of Chicago Press, 38-47.

Titzmann, Peter F./Juang, Linda (2018): Jugendliche mit Migrationshintergrund. In: Gniewosz, Burkhard/Titzmann Peter F. (Hrsg.): Handbuch Jugend. Stuttgart: Kohlhammer, 353-370.

Twum-Danso Imoh, Afua/Bourdillon, Michael/Meichsner, Sylvia (2019): Introduction: Exploring Children's Lives Beyond the Binary of the Global North and Global South. In: Twum-Danso Imoh, Afua/Bourdillo, Michael/Meichsner, Sylvia (Hrsg.): Global Childhoods Beyond the NorthSouth Divide. London: Plagrave MacMillan, 1-10.

Wihstutz, Anne/Schiwarov, Juliana (2018): Kinder als Sorgende - Anmerkungen aus kindheitssoziologischer Perspektive. In: Lange, Andreas/Reiter, Herwig/Steiner, Christine/Schutter, Sabina (Hrsg.): Handbuch Kindheits- und Jugendsoziologie. Wiesbaden: Springer VS, 1-16.

Zito, Dima (2017): Flüchtlinge als Kinder - Kinderflüchtlinge. In: Ghaderi, Cinur/Eppenstein, Thomas (Hrsg.): Flüchtlinge. Multiperspektivische Zugänge. Wiesbaden: Springer VS, 235256. 


\section{Zur Autor*in}

Jessica Schwittek ist Mitarbeiterin an der Fakultät für Bildungswissenschaften der Universität DuisburgEssen. Ihre Arbeitsschwerpunkte liegen im Bereich der sozialwissenschaftlichen Kindheits- und Jugendforschung. Sie forscht zum Aufwachsen in unterschiedlichen Gesellschaften und im Kontext von Migration und Transnationalität.

\section{Kontakt}

Dr. Jessica Schwittek

Universität Duisburg-Essen

Fakultät für Bildungswissenschaften

Institut für Erziehungswissenschaft

AG Sozialisationsforschung

Universitätsstraße 2

45141 Essen

Tel. +49 2011832607

E-Mail: jessica.schwittek@uni-due.de

URL: https://www.uni-due.de/biwi/koenig/schwittek.php 